\title{
Increased high-sensitivity C-reactive protein, erythrocyte sedimentation rate and lactic acid in stroke patients with internal carotid artery occlusion
}

\author{
Dan Xie, Di Hu, Qin Zhang, Yufang Sun, Jimei Li, Yongbo Zhang
}

Department of Neurology, Beijing Friendship Hospital, Capital Medical University, Beijing, China

Submitted: 18 July 2014

Accepted: 12 September 2014

Arch Med Sci 2016; 12, 3: 546-551

DOI: $10.5114 /$ aoms.2014.47879

Copyright ๑ 2015 Termedia \& Banach

\section{Abstract}

Introduction: Internal carotid artery occlusion (ICAO) causes high annual rates of mortality and morbidity. It has been established that atherosclerosis is the normal cause of ICAO. As the pathogenesis of atherosclerosis may involve blood lipids, inflammatory factors and other biomarkers, the aim of this study was to assess the changes in these biomarkers and investigate the relationship between these biomarkers and the development of ICAO in stroke patients.

Material and methods: A total of 89 ischaemic stroke inpatients with ICAO (ICAO group) and 89 without ICAO (control group) were studied, retrospectively. The serum was collected from each patient on the $3^{\text {rd }}$ day of admission, to measure the lipid parameters and biomarkers, e.g. high-sensitivity C-reactive protein (hs-CRP), erythrocyte sedimentation rate (ESR), and lactic acid (LA). Histories were taken including age, gender, smoking history, and disease history. Additional analysis was carried out to compare between the genders and evaluate the association between certain biomarkers and ICAO. Results: Among the 89 ICAO cases in this study, the serum levels of hs-CRP, ESR and LA were significantly higher than those in the control group ( $p \leq$ 0.001). No significant differences were found in the mean levels of total cholesterol, triacylglycerol, HDL cholesterol or glucose, or the known risk factors. Gender also had no influence on these biomarkers. Logistic regression analysis indicated that hs-CRP, ESR and LA were significantly associated with ICAO $(p \leq 0.05)$.

Conclusions: These results suggest that hs-CRP, ESR and LA are associated with ICAO in ischaemic stroke patients, but gender has no effect. Therefore, $\mathrm{Hs}-\mathrm{CRP}, \mathrm{ESR}$ and LA may be useful in the early detection of patients with ICAO.

Key words: internal carotid artery occlusion, inflammatory factors, highsensitivity C-reactive protein, erythrocyte sedimentation rate, lactic acid.

\section{Introduction}

Internal carotid artery occlusion (ICAO) is a chronic and progressive health problem. The risk of death is $7 \%$ at 30 days, $13 \%$ at 1 year and $29 \%$ at 5 years after diagnosis of ICAO $[1,2]$. The risk of cerebral infarction and myocardial infarction is also significantly increased during the next 5 years. Subsequent cerebral ischemia predominantly occurs within several days of stroke onset and is associated with the location, but not the severity, of symptomatic steno-occlusion of cerebral arteries [3]. The overall rate of subsequent stroke is $7 \%$ per year for ischaemic stroke ipsilateral to

\author{
Corresponding author: \\ Yongbo Zhang \\ Department of Neurology \\ Beijing Friendship Hospital \\ Capital Medical University \\ \#95 Yongan Road \\ Xicheng District \\ 100050 Beijing, China \\ Phone: +8613552909549 \\ E-mail: ybzhangcn@sina.com
}


the chronically occluded carotid artery [4]. A recent study demonstrated that $45 \%$ of the stroke patients with ICAO died within the next 1.2 years [5]. Currently, vascular imaging such as CT angiography (CTA) and/or magnetic resonance angiography (MRA) are the standard diagnostic tools [6]; however, these imaging techniques are often not immediately available. Despite recent advances in imaging technology, many local hospitals do not have the capacity to perform CTA and/or MRA [7-9].

To search for a less invasive and cheaper method to detect ICAO, inflammatory factors such as high-sensitivity C-reactive protein (hs-CRP) have been established in several prospective studies [10-12]. High-sensitivity CRP is a very sensitive non-specific biomarker contributing to all stages of atherosclerosis [10, 13-15].

The aim of this study was to assess hs-CRP concentrations along with traditional biomarkers including hs-CRP, erythrocyte sedimentation rate (ESR), lactic acid (LA), etc., in stroke patients with ICAO and to compare them with stroke patients without large vessels stenosis. We also evaluated the possibility of using risk factors and serum concentrations of certain biomarkers, as an assistant for clinical diagnosis of ICAO.

\section{Material and methods}

\section{Patients}

This retrospective study included two groups. All cases of ischaemic stroke were confirmed by computed tomography (CT) or magnetic resonance imaging (MRI) brain scans. These patients were then divided into the control group and the ICAO group depending on the presence of ICAO, determined using transcranial Doppler (TCD) in conjunction with either CTA or MRA. The control group consisted of 89 inpatients (55 males and 34 females) with ischaemic stroke, but no ICAO from January 2008 to June 2008, with an average age of $65.51 \pm 11.49$ years (range: $43-88$ years). The ICAO group included 89 ischaemic stroke inpatients (70 males and 19 females) with ICAO from January, 2003 to March, 2008, in which 85 patients had unilateral ICAO (95.5\%) and 4 patients had bilateral ICAO (4.5\%). The average age of the ICAO group was $64.97 \pm 10.32$ years (range: 40-91 years).

Inclusion criteria for all the patients were as follows: 1) patients were hospitalized due to transient cerebral ischaemic attacks or acute cerebrovascular diseases; 2) cerebral infarction was diagnosed according to the World Health Organization criteria [16], and was confirmed by CT or MRI before admission; 3) head CT scans excluded post-infarction haemorrhage during aggravation of the disease; 4) patients received standard treatment such as antiplatelet, volume expansion, anti-hyperglycaemic, antihypertensive, and neuro-protective therapies. Exclusion criteria were: 1) severe renal or hepatic function deficiency, autoimmune diseases, cancer, haematological diseases, and rheumatic diseases; 2) severe trauma, surgery, or any kind of infection within 2 weeks of disease onset; 3) congenital absence of ICA or MCA; 4) severe stroke: a score less than 20 points according to the National Institutes of Health Stroke Scale (NIHSS).

All the patients enrolled were treated as acute ischaemic stroke victims according to the guidelines. Patients took aspirin $300 \mathrm{mg}$ orally for three days and $100 \mathrm{mg}$ daily afterwards. Among the ICAO group, 11 patients took aspirin $100 \mathrm{mg}$ plus clopidogrel $300 \mathrm{mg}$ for 5 days before interventional treatment following the diagnosis of ICAO. Anti-lipaemic and hypoglycaemic treatment were taken appropriately after the diagnosis of hyperlipaemia or hyperglycaemia respectively.

\section{Methods}

Clinical data including patient's age, gender, smoking history, and disease history such as diabetes mellitus, hypertension, hyperlipidaemia, coronary heart disease, and stroke were retrospectively retrieved from the medical records. Informed consent was obtained from all subjects according to the guidelines of our Ethics Committee.

\section{Blood sampling and C-reactive protein assay}

Blood samples were collected in polystyrene tubes between 08:30 and 10:00 am by venepuncture of the antecubital vein on the $3^{\text {rd }}$ day after admission. Serum glucose, triglyceride, high-density lipoprotein (HDL) cholesterol (direct method) and uric acid concentrations were measured by enzymatic colorimetric methods with commercially available kits. A homogeneous assay was used for selective measurement of low-density lipoprotein (LDL) cholesterol in serum. High-sensitivity CRP in the serum was analysed by an immune turbidimetric assay on a MODULAR-P800 analyser (Roche Diagnostics, Germany) with the manufacturer's reagents, as directed, in the laboratory at Beijing Friendship Hospital of Capital Medical University.

\section{Statistical analysis}

Analyses were performed using SPSS 13.0 (SPSS Inc., Chicago, IL, USA). Numerical data with normal distribution were presented as mean and standard deviation, and were analysed using Student's t-test. Numerical data without normal distribution were presented as the median and interquartile range, and were analysed using the 
Wilcoxon test. Categorical data were compared using the $\chi^{2}$ test. Logistic regression analysis was used to evaluate the relationship between lipid parameters or CRP and the development of ICAO. Statistical significance was considered as $p<0.05$. This study was approved by the Ethics Committee of Beijing Friendship Hospital, Capital Medical University, Beijing, China.

\section{Results}

\section{Evaluation of risk factors and biochemical factors}

Demographic characteristics, risk characteristics and biochemical factors of ICAO cases and control patients are presented in Table I. Of the 89 ICAO cases in this study, 70 (78.65\%) were male, and in the control group only 55 (61.80\%) were male. The proportion of male cases in the ICAO group was significantly higher than that in the control group $(p<0.05)$. There were no significant differences in age, hypertension, diabetes mellitus, hyperlipidaemia, historical stroke or current smokers between the ICAO group and the control group. The mean LDL cholesterol level was $2.78 \pm 0.64 \mathrm{mmol} / \mathrm{l}$ in the ICAO group, which was significantly lower than that in the control group $(2.97 \pm 0.61 \mathrm{mmol} / \mathrm{l}, p<0.05)$. The serum levels of hs-CRP, ESR and LA in the ICAO group were sig- nificantly higher than those in the control group $(p \leq 0.001)$. There were no significant differences in the mean levels of total cholesterol, triacylglycerol, HDL cholesterol, glucose or NIHSS between the two groups.

\section{Logistic regression analysis for patients} with ICAO compared with the control group

Logistic regression analysis was used to examine the relative contributions of lipid parameters, glucose, hs-CRP, ESR, LA and other potential risk factors (age, gender, hypertension, diabetes mellitus, hyperlipidaemia, history of stroke and current smokers) to the development of ICAO (Table II). The results showed that male gender, hs-CRP, ESR and lactic acid were significantly associated with ICAO $(p<0.05)$. The levels of total cholesterol, triglyceride, HDL cholesterol, LDL cholesterol, glucose and some other risk factors (age, hypertension, diabetes mellitus, hyperlipidaemia, history of stroke, current smokers) were not discriminating indicators of the presence of ICAO (Table II).

\section{Comparing the levels of hs-CRP, ESR} and lactic acid in the same gender between two groups

Since male gender was significantly associated with ICAO, we compared the levels of hs-CRP,

Table I. Risk characteristics and biochemical factors in both groups

\begin{tabular}{|c|c|c|c|}
\hline Parameter & ICAO cases $(n=89)$ & Control $(n=89)$ & Value of $p$ \\
\hline Age, mean \pm SD [years] & $64.97 \pm 10.32$ & $65.51 \pm 11.49$ & 0.742 \\
\hline Sex (male/female) & $70 / 19^{*}$ & $55 / 34$ & 0.014 \\
\hline Hypertension, $n$ (\%) & $67(75.3)$ & $63(70.8)$ & 0.501 \\
\hline Diabetes mellitus, $n(\%)$ & $25(28.1)$ & $32(35.9)$ & 0.262 \\
\hline Hyperlipidaemia, $n$ (\%) & $23(25.8)$ & $19(21.3)$ & 0.481 \\
\hline Historical stroke, $n$ (\%) & $37(41.6)$ & $26(29.2)$ & 0.086 \\
\hline Current smokers, $n$ (\%) & $48(53.9)$ & $37(41.6)$ & 0.064 \\
\hline Total cholesterol, mean \pm SD $[\mathrm{mmol} / \mathrm{l}]$ & $4.77 \pm 0.92$ & $4.95 \pm 0.92$ & 0.202 \\
\hline Triacylglycerol, mean \pm SD $[\mathrm{mmol} / \mathrm{l}]$ & $1.71 \pm 0.98$ & $1.55 \pm 0.70$ & 0.203 \\
\hline HDL cholesterol, mean \pm SD $[\mathrm{mmol} / \mathrm{l}]$ & $0.96 \pm 0.25$ & $1.03 \pm 0.26$ & 0.102 \\
\hline LDL cholesterol, mean \pm SD $[\mathrm{mmol} / \mathrm{l}]$ & $2.78 \pm 0.64^{*}$ & $2.97 \pm 0.61$ & 0.044 \\
\hline Glucose, mean \pm SD $[\mathrm{mmol} / \mathrm{l}]$ & $5.95 \pm 1.78$ & $5.92 \pm 1.97$ & 0.927 \\
\hline Hs-CRP, mean \pm SD $[\mathrm{mmol} / \mathrm{l}]$ & $5.31 \pm 4.25^{\dagger}$ & $2.54 \pm 3.32$ & $<0.001$ \\
\hline $\mathrm{ESR}$, mean $\pm \mathrm{SD}[\mathrm{mm} / \mathrm{h}]$ & $19.69 \pm 13.42^{\dagger}$ & $13.63 \pm 8.90$ & 0.001 \\
\hline Lactic acid, mean \pm SD $[\mathrm{mmol} / \mathrm{l}]$ & $3.18 \pm 0.68^{\dagger}$ & $2.82 \pm 0.66$ & $<0.001$ \\
\hline NIHSS, mean \pm SD & $3.92 \pm 4.23$ & $2.73 \pm 2.57$ & 0.0624 \\
\hline
\end{tabular}

Data are shown as mean $\pm S D$, frequency as a number (\%). ${ }^{*} p<0.05$ for differences from control subjects. ${ }^{\dagger} p \leq 0.001$ for differences from control subjects. 
Table II. Logistic regression analysis results for patients with ICAO as compared with the controls

\begin{tabular}{|c|c|c|c|c|}
\hline \multirow[t]{2}{*}{ Predictive variables } & \multirow[t]{2}{*}{ Odds ratio } & \multicolumn{2}{|c|}{$95 \%$ confidence limits of odds ratio } & \multirow[t]{2}{*}{ Value of $p$} \\
\hline & & Lower & Upper & \\
\hline Age & 1.004 & 0.969 & 1.041 & 0.825 \\
\hline Male & 2.728 & 1.055 & 7.058 & 0.038 \\
\hline Hypertension & 1.376 & 0.572 & 3.311 & 0.476 \\
\hline Diabetes mellitus & 0.632 & 0.275 & 1.452 & 0.280 \\
\hline Hyperlipidaemia & 1.724 & 0.662 & 4.489 & 0.264 \\
\hline Historical stroke & 1.710 & 0.781 & 3.747 & 0.180 \\
\hline Current smokers & 0.940 & 0.449 & 1.970 & 0.870 \\
\hline Total cholesterol & 0.765 & 0.261 & 2.244 & 0.626 \\
\hline Triglyceride & 0.750 & 0.459 & 1.223 & 0.249 \\
\hline HDL cholesterol & 0.546 & 0.097 & 3.081 & 0.493 \\
\hline LDL cholesterol & 2.929 & 0.596 & 14.395 & 0.186 \\
\hline Glucose & 1.004 & 0.816 & 1.235 & 0.971 \\
\hline $\mathrm{Hs}-\mathrm{CRP}^{*}$ & 0.795 & 0.705 & 0.897 & $<0.001$ \\
\hline$E S R^{*}$ & 0.936 & 0.897 & 0.976 & 0.002 \\
\hline Lactic acid* & 0.230 & 0.110 & 0.478 & $<0.001$ \\
\hline
\end{tabular}

${ }^{*} p<0.05$ was considered as statistically significant.

ESR and LA within the same gender between the two groups (Table III). The results showed that the levels of hs-CRP, ESR and LA in the ICAO group were significantly higher than those in the control group in males $(p \leq 0.002)$. The level of hs-CRP in the ICAO group was also significantly higher than that in the control group in females ( $p \leq 0.002$ ). There were no significant differences in the levels of hs-CRP, ESR or LA between the male and female cases in the ICAO group.

\section{Discussion}

Ischaemic stroke has become the first cause of death in China. Intracranial atherosclerotic disease (ICAS) causes about $33 \%$ to $54 \%$ of all ischaemic strokes in Asia $[17,18]$. Due to serious medical and economic consequences of ischaemic stroke for the whole society, the identification of biomarkers for ICAO could therefore provide early identification and recognition of high-risk patients for the development of ICAO. These biomarkers could also be used as one of the diagnostic criteria for the application of other highly sensitive and specific, but at the same time expensive and invasive, diagnostic procedures, such as angiography. Because of limited medical expense and long waiting periods for CTA or MRA, identification of novel biochemical markers indicating early stages of ICAO is of great clinical importance. The results of our study showed that patients with ICAO had higher hs-CRP levels, higher serum concentration of ESR and higher LA compared to the control group.

C-reactive protein, an indicator of inflammation and a marker of atherosclerosis [17, 19, 20], is associated with ischaemic attacks in patients with

Table III. Levels of hs-CRP, ESR and LA among cases and control subjects in male and female subjects

\begin{tabular}{|c|c|c|c|c|}
\hline \multirow[t]{2}{*}{ Parameter } & \multicolumn{2}{|c|}{ Male } & \multicolumn{2}{|c|}{ Female } \\
\hline & $\begin{array}{l}\text { ICAO cases } \\
(n=70)\end{array}$ & $\begin{array}{l}\text { Controls } \\
(n=55)\end{array}$ & $\begin{array}{l}\text { ICAO cases } \\
(n=19)\end{array}$ & $\begin{array}{l}\text { Controls } \\
(n=34)\end{array}$ \\
\hline Hs-CRP, mean \pm SD $[\mathrm{mmol} / \mathrm{l}]$ & $5.81 \pm 4.82^{\text {at }}$ & $2.83 \pm 3.79$ & $7.27 \pm 6.41^{\mathrm{dt}}$ & $1.84 \pm 2.70$ \\
\hline $\mathrm{ESR}$, mean $\pm \mathrm{SD}[\mathrm{mm} / \mathrm{h}]$ & $27.30 \pm 22.12^{\mathrm{bt}}$ & $13.11 \pm 11.36$ & $16.38 \pm 7.70$ & $11.35 \pm 8.80$ \\
\hline $\mathrm{LA}$, mean $\pm \mathrm{SD}[\mathrm{mmol} / \mathrm{l}]$ & $3.33 \pm 0.98^{\mathrm{ct}}$ & $2.78 \pm 0.57$ & $3.27 \pm 0.60$ & $2.85 \pm 0.83$ \\
\hline
\end{tabular}

Data are shown as mean $\pm S D$, frequency as a number (\%). ${ }^{a} 0.00155,{ }^{b} 0.00138,{ }^{c} 0.00124,{ }^{d} 0.00019 .{ }^{\dagger} p \leq 0.002$ for differences from control subjects. 
ischaemic stroke [21-26]. It has been reported that serum hs-CRP levels correlate with morphological features of rapidly progressive carotid atherosclerosis, suggesting that hs-CRP is a sensitive marker for the presence of active atherosclerotic disease [27]. In addition, in a case-control study of 600 ischaemic stroke patients and 600 matched controls, serum CRP levels were significantly higher for all ischaemic subtypes than the controls, both in the acute phase and at 3-month follow-up [28]. Rost et al. found that elevated serum CRP levels could predict the risk of future ischaemic stroke in elderly patients [23]. Furthermore, increased hsCRP levels have been found to be associated with the presence of macrophages and $T$ lymphocytes in the plaque, which reflects instability of the plaque that can lead to an ischaemic event [26]. In the present study, we also found that ischaemic stroke patients with ICAO had significantly higher hs-CRP levels than controls, further suggesting that hs-CRP is a sensitive inflammation marker for the presence of ICAO in ischaemic stroke patients.

Evidence suggested that inflammation factors played a very important role in the process of atherosclerosis. The obtained results confirmed ESR as an inflammatory marker in the identification of patients with unstable plaques [23]. Puz et al. [29] investigated 65 patients with ICA stenosis $\geq 50 \%$ and found that ESR and hs-CRP concentrations showed a statistically significant difference from those without stenosis. However, since ESR is a nonspecific marker of inflammation and can be affected by other factors, the results must be used along with other clinical findings. In our study we analysed stroke patients who displayed multiple risk factors for atherosclerosis such as hypertension, diabetes mellitus, and smoking. Our results did not reveal a relationship between the above-mentioned risk factors and ICAO. This may be due to the fact that these factors presented a pre-existing pro-inflammatory/pro-coagulant condition, which may, at least in part, contribute to the increase in ESR values soon after the stroke [30].

As the final product of glycolysis, the concentration of LA is an indicator of the oxidative stress in the human body. When tissues or organs undergo hypoxia, LA concentration increases correspondingly. Studies have shown that real-time monitoring of LA concentration in critically ill patients provides reliable evidence for prognosis and an early warning index [31]. For example, in the case of lactic acidosis, the damage commonly results from perfusion of blood after hypoxia, triggering hyper-secretion of catecholamine. This in turn reduces the capacity of the organ to recover from dysfunction, as implicated in congenital pyruvic dehydrogenase dysfunction. Furthermore, the development of inflammation is also correlated with raised levels of LA. In this study, the concentra- tion of LA in the ICAO group was significantly increased compared to the control group. This may be attributed to the persistence of post-hypoxia perfusion in the hindbrain or artery plaque inflammation. However, further studies investigating the underlying pathology are essential.

Our data indicated that the male gender had a higher incidence of ICAO. However, males normally had a higher percentage of smoking history; therefore, we do not view gender as an independent risk factor. Others have recently demonstrated gender differences in the rates of extra-/intracranial vessel disease; but other factors such as age, race, and stroke severity should also be considered [32]. Through clinical research using a large sample size, Arnold et al. [33] established that there was no gender difference in the rates of middle cerebral artery occlusion, ICAO, and clinical outcome after intra-arterial thrombolysis. While physician bias is one potential explanation, further analysis of this question is beyond the scope of this report. Moreover, we considered the possibility that the results would be affected by medicine, which was metabolized through enzymes of the liver. Since there were no significant differences between the two groups in terms of various risk factors, drug effects should be approximately the same for the two groups. Future studies might be conducted to investigate the effect of drugs in the ICAO group.

In conclusion, our findings showed that the levels of selected inflammatory markers were elevated in patients with ICAO. Hs-CRP, ESR and LA, as biochemical risk markers, may be useful in the detection of patients with ICAO.

\section{Acknowledgments}

Funding for this study was provided by the National Natural Science Foundation of China and Beijing Municipal Health System High-Level Technician Cultivation Project. We thank Dr Zanmin Song for comments that greatly improved the manuscript. We also thank the two anonymous reviewers for their critical and constructive comments.

\section{Conflict of interest}

The authors declare no conflict of interest.

\section{References}

1. Flaherty ML, Flemming KD, McClelland R, Jorgensen NW, Brown RD, Jr. Population-based study of symptomatic internal carotid artery occlusion: incidence and longterm follow-up. Stroke 2004; 35: e349-52.

2. Paraskevas KI, Mikhailidis DP, Liapis CD. Internal carotid artery occlusion: association with atherosclerotic disease in other arterial beds and vascular risk factors. Angiology 2007; 58: 329-35. 
3. Kang J, Kim N, Oh CW, et al. Symptomatic steno-occlusion of cerebral arteries and subsequent ischemic events in patients with acute ischemic stroke. J Stroke Cerebrovasc Dis 2014; 23: e347-53.

4. Young FB, Weir CJ, Lees KR, Committee GITS, Investigators. Comparison of the National Institutes of Health Stroke Scale with disability outcome measures in acute stroke trials. Stroke 2005; 36: 2187-92.

5. Paciaroni M, Caso V, Venti M, et al. Outcome in patients with stroke associated with internal carotid artery occlusion. Cerebrovasc Dis 2005; 20: 108-13.

6. lezzi R, Santoro M, Marano R, et al. Low-dose multidetector CT angiography in the evaluation of infrarena aorta and peripheral arterial occlusive disease. Radiology 2012; 263: 287-98.

7. Choi JC, Kang SY, Kang JH, Ko YJ, Bae JM. Are in-hospital delays important obstacles in thrombolytic therapy following acute ischemic stroke? J Clin Neurol 2007; 3: 71-8.

8. Choi JY, Kim EJ, Kim HS, et al. The missing button sign as a tool for detecting proximal internal carotid artery occlusion. J Clin Neurosci 2010; 17: 1506-9.

9. Latchaw RE, Alberts MJ, Lev MH, et al. Recommendations for imaging of acute ischemic stroke: a scientific statement from the American Heart Association. Stroke 2009; 40: 3646-78.

10. Libby P, Ridker PM. Inflammation and atherosclerosis: role of C-reactive protein in risk assessment. Am J Med 2004; 116 Suppl. 6A: 9S-16S.

11. Koenig W. Predicting risk and treatment benefit in atherosclerosis: the role of C-reactive protein. Int J Cardio 2005; 98: 199-206.

12. Rifai N, Ridker PM. High-sensitivity C-reactive protein: a novel and promising marker of coronary heart disease. Clin Chem 2001; 47: 403-11.

13. Flegar-Mestric Z, Vrhovski-Hebrang D, Preden-Kerekovic V, et al. C-reactive protein level in severe stenosis of cerebral arteries. Cerebrovasc Dis 2007; 23: 430-4.

14. Verma S, Szmitko PE, Ridker PM. C-reactive protein comes of age. Nature clinical practice. Cardiovasc Med 2005; 2: 29-36.

15. Whicher J, Biasucci L, Rifai N. Inflammation, the acute phase response and atherosclerosis. Clin Chem Lab Med 1999; 37: 495-503.

16. Li JJ, Fang $\mathrm{CH}$. C-reactive protein is not only an inflammatory marker but also a direct cause of cardiovascular diseases. Med Hypotheses 2004; 62: 499-506.

17. Liu M, Wu B, Wang WZ, Lee LM, Zhang SH, Kong LZ. Stroke in China: epidemiology, prevention, and management strategies. Lancet Neurol 2007; 6: 456-64.

18. Wong KS, Li H, Chan YL, et al. Use of transcranial Doppler ultrasound to predict outcome in patients with intracranial large-artery occlusive disease. Stroke 2000; 31: 2641-7.

19. Bassuk SS, Rifai N, Ridker PM. High-sensitivity C-reactive protein: clinical importance. Curr Probl Cardio 2004; 29: 439-93.

20. Koyama K, Yoneyama K, Mitarai T, et al. Association between inflammatory biomarkers and thin-cap fibro atheroma detected by optical coherence tomography in patients with coronary heart disease. Arch Med Sci 2015; 11: 505-12.

21. Alvarez Garcia B, Ruiz C, Chacon P, Sabin JA, Matas M High-sensitivity $C$-reactive protein in high-grade carotid stenosis: risk marker for unstable carotid plaque. J Vasc Surg 2003; 38: 1018-24.
22. Christensen H, Boysen G. C-reactive protein and white blood cell count increases in the first 24 hours after acute stroke. Cerebrovasc Dis 2004; 18: 214-9.

23. Rost NS, Wolf PA, Kase CS, et al. Plasma concentration of C-reactive protein and risk of ischemic stroke and transient ischemic attack: the Framingham study. Stroke 2001; 32: 2575-9.

24. Song IU, Kim JS, Kim YI, Lee KS, Jeong DS, Chung SW. Relationship between high-sensitivity C-reactive protein and clinical functional outcome after acute ischemic stroke in a Korean population. Cerebrovasc Dis 2009; 28: 545-50.

25. Thomas GN, Chen XY, Lin JW, et al. Middle cerebral artery stenosis increased the risk of vascular disease mortality among type 2 diabetic patients. Cerebrovasc Dis 2008; 25: 261-7.

26. Wong KS, Ng PW, Tang A, Liu R, Yeung V, Tomlinson B. Prevalence of asymptomatic intracranial atherosclerosis in high-risk patients. Neurology 2007; 68: 2035-8

27. Schillinger M, Exner M, Mlekusch W, et al. Inflammation and carotid artery: risk for atherosclerosis study (ICARAS). Circulation 2005; 111: 2203-9.

28. Ladenvall C, Jood K, Blomstrand C, Nilsson S, Jern C, Ladenvall $P$. Serum $C$-reactive protein concentration and genotype in relation to ischemic stroke subtype. Stroke 2006; 37: 2018-23

29. Puz P, Lasek-Bal A, Ziaja D, Kazibutowska Z, Ziaja K. Inflammatory markers in patients with internal carotid artery stenosis. Arch Med Sci 2013; 9: 254-60.

30. Singh AS, Atam V, Yathish BE, Das L, Koonwar S. Role of erythrocyte sedimentation rate in ischemic stroke as an inflammatory marker of carotid atherosclerosis. J Neurosci Rural Pract 2014; 5 : 40-5.

31. Lee JR, Choi HR. Validation of a modified early warning score to predict ICU transfer for patients with severe sepsis or septic shock on general wards. J Korean Acad Nurs 2014; 44: 219-27.

32. Flaherty ML, Kissela B, Khoury JC, et al. Carotid artery stenosis as a cause of stroke. Neuroepidemiology 2013; 40: 36-41.

33. Arnold M, Kappeler L, Nedeltchev K, et al. Recanalization and outcome after intra-arterial thrombolysis in middle cerebral artery and internal carotid artery occlusion: does sex matter? Stroke 2007; 38: 1281-5. 